\title{
Coherent emission from large arrays of discrete Josephson junctions
}

F. Song (宋凤斌), F. Müller, R. Behr, and A. M. Klushin

Citation: Appl. Phys. Lett. 95, 172501 (2009);

View online: https://doi.org/10.1063/1.3253417

View Table of Contents: http://aip.scitation.org/toc/apl/95/17

Published by the American Institute of Physics

\section{Articles you may be interested in}

Compact tunable sub-terahertz oscillators based on Josephson junctions

Applied Physics Letters 98, 142506 (2011); 10.1063/1.3576910

Superlattice electronic devices as high-performance oscillators between $60-220 \mathrm{GHz}$ Applied Physics Letters 96, 072101 (2010); 10.1063/1.3324697

Characteristics of terahertz radiation emitted from the intrinsic Josephson junctions in high- $T_{\mathrm{C}}$ superconductor $\mathrm{Bi}_{2} \mathrm{Sr}_{2} \mathrm{CaCu}_{2} \mathrm{O}_{8+\delta}$

Applied Physics Letters 95, 232511 (2009); 10.1063/1.3269996

Continuous $30 \mu W$ terahertz source by a high- $\mathrm{T}_{\mathrm{C}}$ superconductor mesa structure Applied Physics Letters 103, 182601 (2013); 10.1063/1.4827094

Terahertz emission and detection both based on high- $\mathrm{T}_{\mathrm{C}}$ superconductors: Towards an integrated receiver Applied Physics Letters 102, 092601 (2013); 10.1063/1.4794072

Effect of thermal inhomogeneity for terahertz radiation from intrinsic Josephson junction stacks of $\mathrm{Bi}_{2} \mathrm{Sr}_{2} \mathrm{CaCu}_{2} \mathrm{O}_{8+\delta}$

Applied Physics Letters 100, 242603 (2012); 10.1063/1.4727899

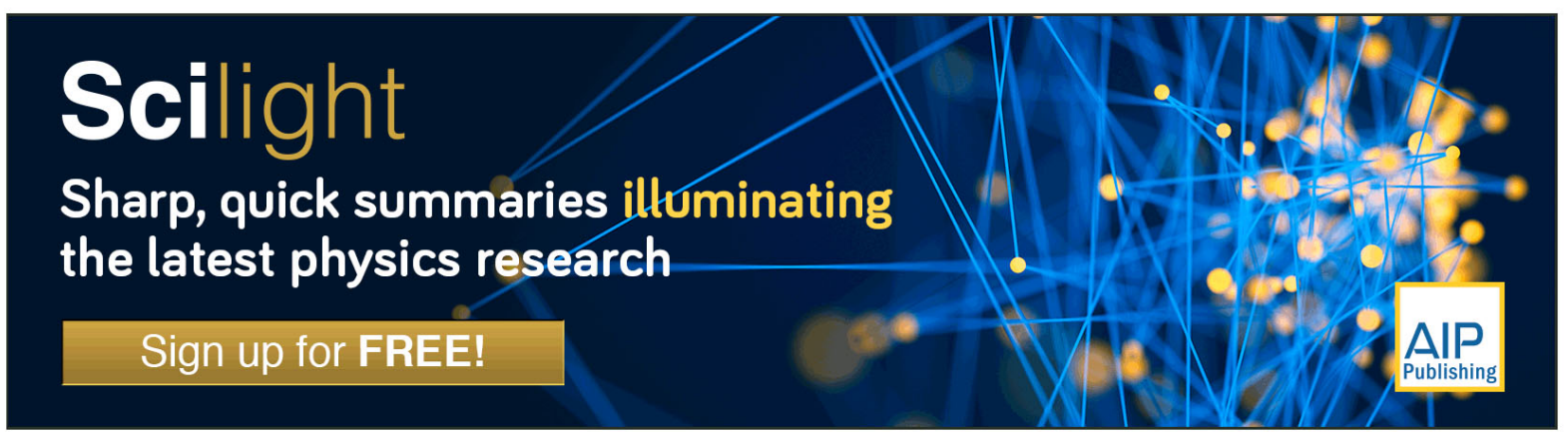




\title{
Coherent emission from large arrays of discrete Josephson junctions
}

\author{
F. Song (宋凤斌), ${ }^{1,2}$ F. Müller, ${ }^{3}$ R. Behr, ${ }^{3}$ and A. M. Klushin ${ }^{1, a)}$ \\ ${ }^{1}$ Institute of Bio- and Nanosystems and JARA-Fundamentals of Future Information Technology, \\ Forschungszentrum Jülich, D-52425 Jülich, Germany \\ ${ }^{2}$ Department of Electronics, Nankai University, 300017 Tianjin, People's Republic of China \\ ${ }^{3}$ Physikalisch-Technische Bundesanstalt, 38116 Braunschweig, Germany
}

(Received 9 September 2009; accepted 1 October 2009; published online 26 October 2009)

\begin{abstract}
We observed coherent emission at the frequency of $76 \mathrm{GHz}$ from a large-area array of 7500 niobium junctions embedded in the quasioptical resonator at liquid helium temperature. The maximal detected power of $2 \mu \mathrm{W}$ was measured by a room temperature superheterodyne receiver. To explain the coherent behavior of the array of Josephson junctions, electromagnetic field simulations were performed. Considering the substrate as a dielectric resonator antenna, the coherent emission was excited if the locations of each subarray coincided with the positions of the maximum electric field intensity in the substrate resonance mode. (C) 2009 American Institute of Physics.
\end{abstract}

[doi:10.1063/1.3253417]

Although compact and powerful terahertz semiconductor continuous-wave radiation sources have been extensively investigated for several decades because of their wide variety of applications, ${ }^{1}$ there is still a frequency gap approximately from 0.5 to $1.5 \mathrm{THz}{ }^{2,3}$ Fortunately Josephson junctions can be ideal voltage-to-frequency transducers filling this gap with the bias voltage of several millivotls, which has been confirmed by Ozyuzer et al. ${ }^{4}$ utilizing coherent intrinsic Josephson junctions in the layered high-temperature superconductor $\mathrm{Bi}_{2} \mathrm{Si}_{2} \mathrm{CaCu}_{2} \mathrm{O}_{8}$.

Self-emission of arrays of discrete Josephson junctions has been under investigation for many years. ${ }^{5}$ Substantial progress in the technology of low-temperature superconductor (LTS) and high-temperature superconductor (HTS) Josephson junctions makes this approach very promising for the development of the radiation sources in terahertz frequency band. For example, an increase of the characteristic frequency in niobium junctions with $\mathrm{Nb}_{x} \mathrm{Si}_{1-x}$ barrier was presented by Olaya et al., ${ }^{6}$ which makes it possible to use these junctions as radiation sources at the frequencies limited by the superconductor gap of niobium. Recently, Divin et al. ${ }^{7}$ demonstrated that ac Josephson effect in HTS bicrystal junctions can be observed up to $5 \mathrm{THz}$. To increase the emission power, coherently oscillating arrays of discrete Josephson junctions should be used. ${ }^{8}$ Important prerequisites for synchronization of Josephson junctions are a small spread of the parameters of the junctions and an effective microwave coupling between the junctions and to the external microwave system.

In this letter, we present an approach to obtain a coherent emission from a large area discrete array of Josephson junctions with the assistance of both their substrate and a quasioptical resonator. We propose to use the substrate as a dielectric resonator antenna ${ }^{9,10}$ gathering the emission power from Josephson junctions and improving their coupling to the external quasioptical resonator. Limited by the fabrication technology of HTS junctions, we chose a series array of $\mathrm{Nb}-\mathrm{Al}_{2} \mathrm{O}_{3}-\mathrm{Al}-\mathrm{Al}_{2} \mathrm{O}_{3}-\mathrm{Nb}$ (SINIS) junctions to verify our idea at a gigahertz frequency.

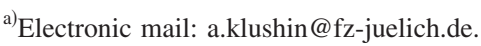

Our measurement system is schematically shown in Fig. 1. Josephson emission was detected using a superheterodyne radiometer with intermediate frequency bandwidth of $1.4 \mathrm{GHz} .{ }^{11}$ The sample was embedded in a quasioptical resonator $^{12}$ in which a horn antenna was used instead of the hemispherical mirror. The power of intermediate frequency of the double side-band receiver versus the voltage applied to the array was monitored by either a lock-in detector or the HP 8592B spectrum analyzer.

The series array of 7500 SINIS Josephson junctions ${ }^{13}$ contained six meandering subarrays of 1250 junctions placed in ten per group as it is shown at the right of Fig. 1. Special thin-film conductor tabs allowed us to independently dc-bias each separate subarray and measure voltage across it. The array occupying the area of $4.6 \times 5 \mathrm{~mm}^{2}$ was fabricated on a $10 \times 10 \times 0.38 \mathrm{~mm}^{3}$ silicon substrate.

From the measurement of current-voltage $(I-V)$ curve of the entire array without irradiation shown in Fig. 2, curve a, the critical current $I_{c}$ was $1.9 \mathrm{~mA}$, the average normal resistance of a single junction $R_{n}=0.032 \Omega$. Therefore, for each junction the average characteristic voltage $V_{c}$ was $0.06 \mathrm{mV}$ and the characteristic frequency of $29 \mathrm{GHz}$.

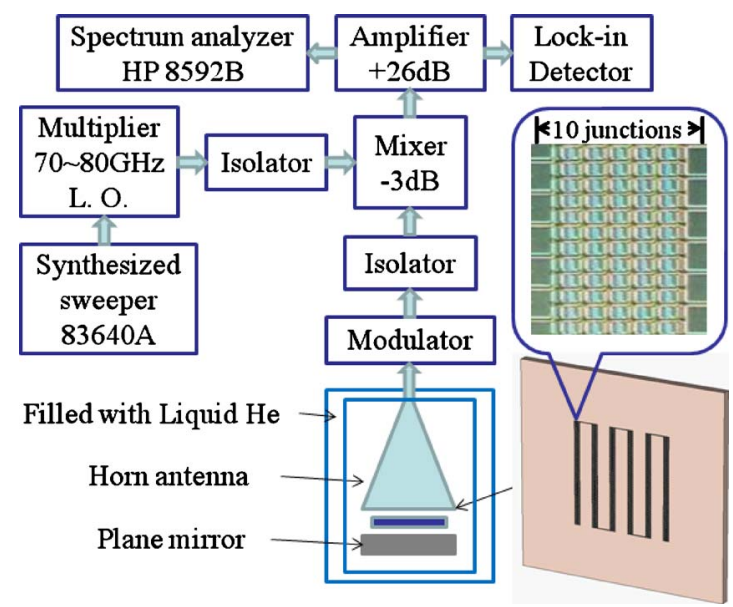

FIG. 1. (Color online) Schematic diagram of the measurement setup. Right side shows schematically a large series array of $N=7500$ SINIS Josephson junctions, equally divided into six subarrays on the substrate. Above it, the enlarged view is a photo of a small part of the meandering structure. 


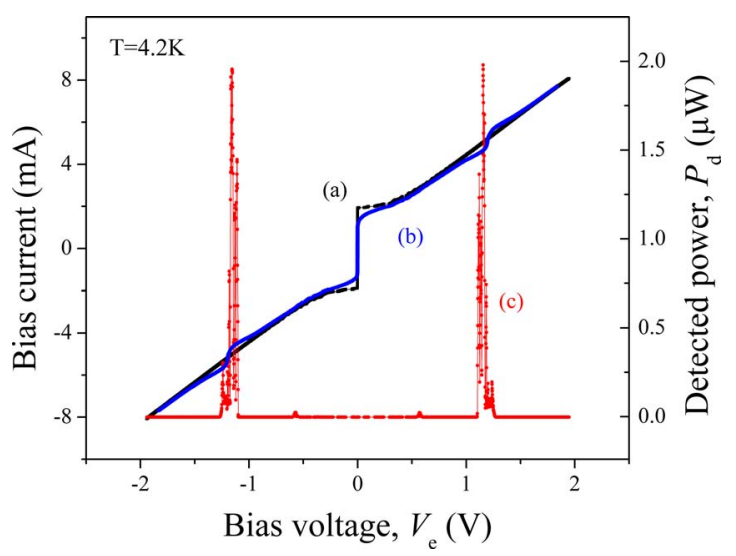

FIG. 2. (Color online) The $I-V$ curve of the series array without irradiation (curve a); the $I-V$ curve of the series array with irradiation at $75.2 \mathrm{GHz}$ (curve b); dependence of emission power $P(V)$ from the series array on $V_{e}$ measured by lock-in detector (curve c).

With an external irradiation at $75.2 \mathrm{GHz}$, Shapiro steps were observed (Fig. 2, curve b) by adjusting the cavity length of the external quasioptical resonator and the distance between sample and plane mirror. In this case, the optimum coupling of the substrate to the external resonator was realized.

When we swept the bias current of the series array, the emitted power was registered by the lock-in detector. Several symmetrical peaks were observed at different bias voltages $V_{e}$ as it is shown in Fig. 2, curve c. The maximum detected power $P_{d} \cong 2 \mu \mathrm{W}$ was measured at $V_{e}=1.156 \mathrm{~V}$. According to the theory, the maximum emitted power $P_{\max }$ from a series array of $N$ junctions in the case of optimum impedance matching can be estimated by the following formula: ${ }^{8}$

$$
P_{\max }=N I_{c}^{2} R_{n} / 8 .
$$

The $P_{\max }$ could be $108 \mu \mathrm{W}$ would our whole array emit coherently. We thus attained the emission efficiency of about $2 \%$.

Supposing that identical junctions are connected in series, central emission frequency $f_{J}$ can be evaluated from the Josephson equation:

$$
f_{J}=V_{e} / N \Phi_{0},
$$

where $\Phi_{0}$ is the flux quantum. According to Eq. (2) the frequency $f_{J}$ was equal to $74.5 \mathrm{GHz}$.

At the same $V_{e}=1.156 \mathrm{~V}$ a major emission peak at $f_{J}$ equal to $76.0 \mathrm{GHz}$ was also observed by the spectrum analyzer with local oscillator frequency $f_{\mathrm{LO}}=76.8 \mathrm{GHz}$ and the resolution bandwidth $3 \mathrm{MHz}$. It is shown in the insert of Fig. 3. The $-3 \mathrm{~dB}$ linewidth of the emission peak was equal to $9 \mathrm{MHz}$ and limited by many factors including the stability of our bias current source. Within the $3 \mathrm{MHz}$ resolution bandwidth at $76.0 \mathrm{GHz}$ the emission power was $0.34 \mu \mathrm{W}$.

There is a nearly $2 \%$, difference between the values of the calculated $(74.5 \mathrm{GHz})$ and the directly observed $(76$ $\mathrm{GHz}$ ) central emission frequency. To explain this difference we should take into account the spread of the junctions' normal resistances in the series array. With the common bias current, the spread of voltage drop on the junctions appeared due to the spread of the $R_{n}$. Our data documented that the voltage applied to the most junctions corresponds to $f_{J}$ $=76 \mathrm{GHz}$.

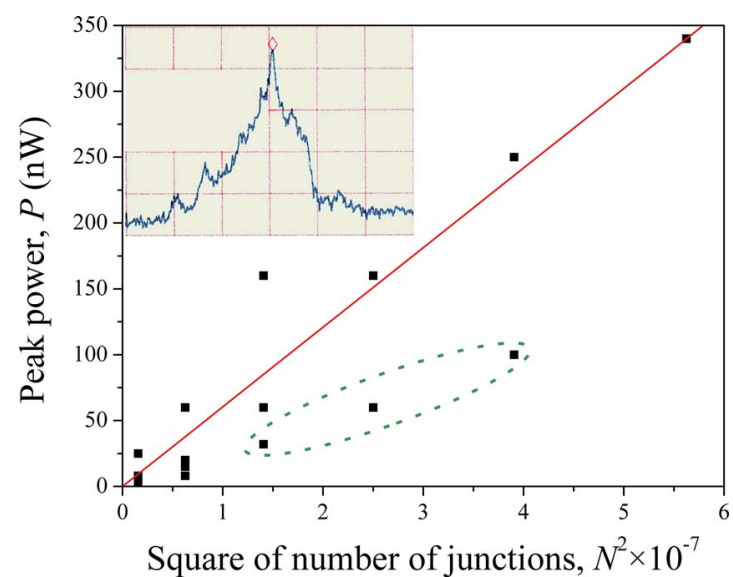

FIG. 3. (Color online) Emission power ( $\square$ ) within $3 \mathrm{MHz}$ resolution bandwidth of spectrum analyzer at peaks as a function of $N^{2}$. The line is leastsquares fit to the experimental data points, except for the three points surrounded by the dotted curve which came from subarrays with large difference in the resistance. Inset shows the emission peak observed by spectrum analyzer at $V_{e}=1.156 \mathrm{~V}$. Scale: axis $x$ : $100 \mathrm{MHz} /$ div., axis $y$ : 10 dB/div.

To prove the existence of the mutual phase locking of junctions in the array, all possible combinations of the six subarrays were dc biased and the detected power was recorded by the spectrum analyzer. The detected peak power was a nearly linear function of the square of the number of junctions $N$ in the measured subarray combination. This observed linear dependence is in accordance with the theory and experimental results reported previously. ${ }^{4,8}$

To illustrate the coherent behavior of discrete junctions in the explored series array, electromagnetic field simulations were performed with the CST (Computer Simulation Technology) microwave studio software. ${ }^{14}$ A model with experimental parameters of the series array and of the substrate, including dimensions and dielectric constant $\varepsilon_{\mathrm{r}}=11.9$, was constructed. Josephson junctions were modeled to be discrete ports with equal resistances $R_{n}$ and with phase-locked alternating currents (ac). The meandering superconductive connections of Josephson junctions were modeled by perfect metal. Considering the substrate as a dielectric resonator, the chosen resonance mode could be excited if the location of each subarray coincided with the position of the maximum electric field intensity. As shown in Fig. 4, the resonance mode at $76 \mathrm{GHz}$ was stimulated by the model array of discrete ports. This proves the ac power from the junctions (ports) was coupled into the substrate, accumulated by its resonance [Fig. 4(a)], and then emitted into the external quasioptical resonator forming standing waves. In Fig. 4(b), the cross section of the $y$ axis component of the electric field intensity shows coupling of the resonance mode of the substrate and the mode of the quasioptical resonator.

In conclusion, utilizing the intrinsic resonant mode of the substrate embedded in the open resonator, a coherent self-emission from the series array of LTS Josephson junctions was directly observed by the spectrum analyzer at 76 GHz. The maximum power measured by the lock-in detector was equal to $2 \mu \mathrm{W}$, which corresponds to the emission efficiency of $2 \%$. We believe, discrete junction arrays working with larger emission efficiency can be designed on optimized substrates with suitable resonant modes at terahertz frequencies by simulations. It is important to note that our quasiop- 

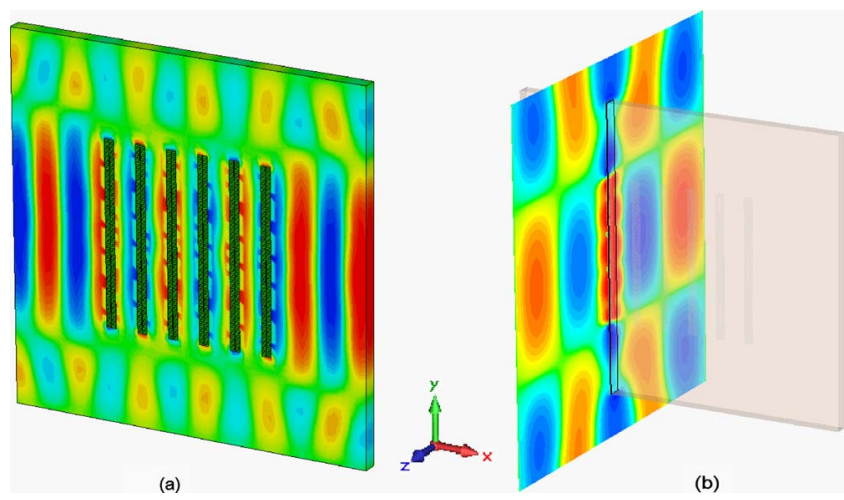

FIG. 4. (Color online) The resonance mode of the silicon substrate at 76 $\mathrm{GHz}$ is stimulated by the array of discrete ports with $R_{n}$. (a) Distribution of $y$ axis electric field intensity on the substrate which serves as a dielectric resonator antenna. (b) Cross-section of the $y$ axis component of the electric field intensity shows coupling of the resonance mode of the substrate and the mode of the quasioptical resonator.

tical coupling method can be extended up to terahertz frequencies. With HTS Josephson junctions operating at liquid nitrogen temperature such arrays will be a promising approach to continuous wave terahertz sources.

The author Fengbin Song is supported by the State Scholarship Fund organized by CSC (China Scholarship
Council). The authors would like to thank G. Panaitov for the help by the modification of the samples, N. Klein for stimulating discussions and valuable support, and A. Braginski for a careful reading of the paper and useful suggestions.

${ }^{1}$ M. Tonouchi, Nat. Photonics 1, 97 (2007).

${ }^{2}$ R. Kleiner, Science 318, 1254 (2007).

${ }^{3}$ C. Walther, G. Scalari, J. Faist, H. Beere, and D. Ritchie, Appl. Phys. Lett. 89, 231121 (2006).

${ }^{4}$ L. Ozyuzer, A. E. Koshelev, C. Kurter, N. Gopalsami, Q. Li, M. Tachiki, K. Kadowaki, T. Yamamoto, H. Minami, H. Yamaguchi, T. Tachiki, K. E. Gray, W. K. Kwok, and U. Welp, Science 318, 1291 (2007).

${ }^{5}$ M. Darula, T. Doderer, and S. Beuven, Supercond. Sci. Technol. 12, R1 (1999).

${ }^{6}$ D. Olaya, P. Dresselhaus, S. Benz, J. Bjarnason, and E. Grossman, IEEE Trans. Appl. Supercond. 19, 144 (2009).

${ }^{7}$ Y. Y. Divin, O. Y. Volkov, M. V. Liatti, and V. N. Gubankov, IEEE Trans. Appl. Supercond. 13, 676 (2003).

${ }^{8} \mathrm{~K}$. K. Likharev, Dynamics of Josephson Junctions and Circuits (Gordon and Breach, New York, 1986).

${ }^{9}$ Dielectric Resonator Antennas edited by K. M. Luk and K. W. Leung (Research Studies Press Ltd., Hertfordshire, 2003).

${ }^{10}$ D. Kajfez and P. Guillon, Dielectric Resonators (Noble, Atlanta, 1998).

${ }^{11}$ G. Kunkel, R. H. Ono, and A. M. Klushin, Supercond. Sci. Technol. 9, A1 (1996).

${ }^{12}$ A. M. Klushin, M. He, S. L. Yan, and N. Klein, Appl. Phys. Lett. 89, 232505 (2006).

${ }^{13}$ F. Müller, H. Schulze, R. Behr, J. Kohlmann, and J. Niemeyer, Physica C 354, 66 (2001).

${ }^{14} \mathrm{CST}$ microwave studio, available: http://www.cst.com. 